Revista ELectrónica de Investigación y EValuación Educativa
RELIEVE

ISSN: 1134-4032
e-Journal of Educational Research,

Assessment and Evaluation

\title{
Divergencias del alumnado y del profesorado universitario sobre las dificultades para aplicar la evaluación formativa
}

\author{
Differences of students and faculty on the difficulties to implement the formative assessment
}

\author{
Romero-Martín, Rosario ${ }^{(1)}$; Castejón-Oliva, Francisco-Javier ${ }^{(2)}$; López-Pastor, Víctor (3) \\ (1) Universidad de Zaragoza. (2) Universidad Autónoma de Madrid. (3) Universidad de Valladolid
}

\begin{abstract}
Resumen
La evaluación formativa supone un claro cambio en la forma de plantear la evaluación en la docencia universitaria. Su aplicación no está exenta de cierta controversia, y sobre todo de dificultades, que hacen que alumnado y profesorado tengan distintas perspectivas. El objetivo del presente estudio ha sido comprobar las divergencias del alumnado y del profesorado cuando se diseña y se ponen en práctica sistemas de evaluación formativa. Se ha utilizado un cuestionario para el alumnado $(\mathrm{N}=3013)$ y los informes elaborados por el profesorado $(\mathrm{N}=46)$ aplicados durante el curso 2012-13. Los resultados muestran que la evaluación formativa es bastante exigente para el alumnado, aunque también muestran una alta satisfacción del alumnado con la misma, particularmente con la calificación. Un inconveniente que refleja el alumnado parece ser la acumulación de trabajo al final del proceso, lo cual resulta paradójico y requiere de una investigación más profunda en el futuro. El profesorado también muestra una alta satisfacción con este tipo de evaluación, aunque tengan una mayor carga de trabajo. Se observan escasas discrepancias entre los dos colectivos (alumnado y profesorado). En el estudio se proponen recomendaciones de puesta en práctica en cuanto al diseño y la planificación para investigaciones futuras. Las limitaciones se observan en cuanto a la población participante en el estudio, con el fin de que puedan generalizarse los resultados.
\end{abstract}

\section{Palabras clave:}

Evaluación Formativa; Evaluación participativa; Educación Superior; Percepción del estudiante universitario; Percepción del docente

\begin{abstract}
Formative Assessment shows a clear change in how to approach the evaluation of university teaching. The application of Formative Assessment is not without some controversy, and above all difficulties due to students and teachers having different perspectives. The objective of the present study was to check the differences of students and teachers when formative assessment systems are designed and put into practice. A questionnaire was used for students $(\mathrm{N}=3013)$ and a report prepared by the teachers $(\mathrm{N}=46)$. Both were taken from the academic year 201213. The results show that formative assessment is quite demanding for students, but also that students are highly satisfied with it, particularly with grades. A problem that the students reflect seems to be the accumulation of work at the end of the process, which is paradoxical and will requires further research in the future. Teachers also show a high satisfaction with this type of assessment, even if they have a greater workload. There are few differences between the two groups (students and teachers). The study proposes recommendations for implementation in terms of design and planning for future research. The limitations of the research can be seen in terms of participating in the study population, so the results can be generalized.
\end{abstract}

Fecha de recepción 21 de Enero de 2014

Fecha de aprobación 20 de Abril de 2015

Fecha de publicación 23 de Abril de 2015

\section{Keywords:}

Formative Assessment; Participative assessment; Higher Education; Teachers Perception; 
La aplicación de la evaluación formativa en educación superior no está exenta de dificultades para el profesorado y el alumnado. No es solo una cuestión de desconocimiento, también hay resistencias y falta de tradición para poder aplicarla (Capllonch et al., 2009; Pérez et al., 2008). En el presente trabajo vamos a analizar la percepción que el alumnado tiene sobre las desventajas de utilizar sistemas de evaluación formativa en las aulas universitarias.

Para nuestro estudio, consideramos que la evaluación formativa tiene como finalidad mejorar el proceso de aprendizaje del alumnado y la labor docente del profesorado (López-Pastor, 2009). Se ha señalado que este proceso orienta y reorienta el proceso de enseñanza-aprendizaje para que el alumnado logre mejores aprendizajes y resultados académicos (Torrance, 2012), y también se ha señalado su incidencia en el desarrollo de sus competencias y autonomía (Rué, 2009). Black y Wiliam (2009) y Yorke (2003) consideran que la evaluación formativa debe plantearse y ponerse en práctica de forma que verdaderamente cumpla con su intención; por ejemplo, fomentando la auto-regulación e incidiendo en el aprendizaje profundo para el alumnado, lo cual requiere que el profesorado asuma la independencia del alumnado.

Algunos de los estudios que defienden el uso de la evaluación formativa entienden que el alumnado debe estar implicado en la misma (Biggs, 2005; Boud y Falchikov, 2007; Brown y Glasner, 2007; Knight, 2005), pues consideran que hay una relación estrecha entre participación activa del alumnado y mejora en los aprendizajes (Bowden y Marton, 2012; Cano, 2012; Marton y Säljö, 1976). Debido a esta implicación, parece que la evaluación formativa ofrece mejores resultados de aprendizaje y mayor rendimiento académico (Carrillo et al., 2009; Castejón, López-Pastor, Julián y Zaragoza, 2011; Romero-Martín, Fraile-Aranda, López-Pastor y Castejón-Oliva,
2014). La evaluación formativa requiere que haya una retroalimentación adecuada para que el alumnado pueda avanzar en sus aprendizajes; los efectos del feedback en la evaluación formativa han demostrado dicha mejora siempre que se cumplan los preceptos de un buen feedback (Nicol y Macfarlane, 2006): cuando aporta información adecuada, comprensible y que le permita resolver los problemas de su aprendizaje, repercuta en su motivación y mantenga el interés por mejorar en su rendimiento (ver también Burke, 2009; Carless, 2006; Carless, Salter, Yang y Lam, 2010).

En cuanto a las diferentes temáticas o disciplinas, los estudios que han analizado los efectos de la evaluación formativa aportan resultados a tener en cuenta. En estudios de medicina, Krasner, Wimmers, Relan y Drake (2006) demostraron que el uso de la evaluación formativa predice mejor los resultados del alumnado que la evaluación sumativa, que las estrategias que usaron en la evaluación formativa redujeron la tensión y el estrés, y suponen una mejor utilización del tiempo de aprendizaje; algo que no se resuelve en la evaluación sumativa, donde prima lo memorístico, la rendición de cuentas y la medición de resultados finales, es decir, “jugarse todo a una carta”. Por otro lado, la recopilación de estudios experimentales sobre la puesta en práctica de la evaluación formativa en ciencias sociales muestra que sus efectos son mejores que el uso de la evaluación tradicional (Santos, Martínez y López-Pastor, 2009).

Para llegar a conseguir que el alumnado sea responsable de su aprendizaje, una estrategia importante es la puesta en marcha de procesos de evaluación formativa, de autoevaluación y de evaluación entre iguales. En este sentido, Fallows y Chandramohan (2001) señalan que la introducción de la autoevaluación y la evaluación entre iguales proporciona beneficios más que justificados 
para su implementación en la educación superior. Los estudios muestran que el alumnado ofrece garantías de actuar correctamente, aunque en mayor medida con alumnado de últimos cursos. No obstante, también encontramos que el alumnado no siempre participa con el mismo entusiasmo, las divergencias entre la posición del profesorado y del alumnado aparecen, bien por sus reticencias a evaluar a los compañeros, bien por el trabajo que supone realizar esa evaluación (Gijbels y Dochy, 2006).

La aplicación de la evaluación formativa suele generar una mejora en el rendimiento académico del alumnado, particularmente cuando se han planteado unos criterios adecuados para su aplicación (Sadler, 2005). Varios estudios llevados a cabo en la formación inicial del profesorado encuentran mejoras considerables en las calificaciones del alumnado (Castejón et al., 2011; Julián, Zaragoza, Castejón y López-Pastor, 2010; López-Pastor, 2008; López-Pastor, Manrique y Vallés, 2011), aunque también señalan que el alumnado sigue ofreciendo ciertas resistencias a la hora de participar en este tipo de sistemas de evaluación y diverge de lo que plantea el profesorado. Conocer la opinión del alumnado parece, entonces, relevante para aclarar esta aparente paradoja, y avanzar en el conocimiento si además se contrasta con la del profesorado.

Existen estudios que investigan la percepción del alumnado respecto a la utilización de sistemas de evaluación formativa y compartida en educación superior y su influencia en variables como: percepción de aprendizaje, participación y protagonismo en el proceso y carga de trabajo que supone. Un ejemplo es el de Hamodi y López-Pastor (2012), utilizando el grupo de discusión como herramienta de obtención de datos. En este estudio participaron alumnados de último curso y egresados, todos ellos en formación inicial del profesorado. Los autores detectaron que el alumnado no entiende al profesorado cuando plantean la evaluación, particularmente cuando el discurso es que hay que hacer evaluación continua, y lo que terminan haciendo es "jugarse" la asignatura a un examen final a veces unido a un trabajo que hay que aprobar por separado para recibir una calificación media. Es decir, que las divergencias no se manifiestan solo por lo que percibe el alumnado, también por la falta de formación didáctica del profesorado, algo que se había señalado con anterioridad (Fernández, 1989; Tejedor, 1998) y que sigue siendo un punto crítico en la formación inicial del profesorado (De la Calle, 2004; Tonucci, 2010; Trillo, 2005). En esta misma línea se han manifestado otros trabajos (Boussada y De Ketele, 2008). Sin embargo, hay otros estudios que sí muestran una continuidad entre lo aprendido sobre evaluación formativa en su formación inicial y lo que luego ponen en práctica de evaluación formativa en su desarrollo profesional (Hamodi y LópezPastor, 2012).

Por otro lado, el profesorado pone en práctica procesos evaluativos para comprobar los aprendizajes del alumnado, que muchas veces son contradictorios con el discurso institucional (normativa) y personal (formación pedagógica). Así, Palacios y López-Pastor (2013) señalan tres tipos de profesorado: (a) innovador (25\%), con actitudes positivas hacia la evaluación continua y formativa; (b) tradicional (26\%), con actitudes no favorables a los sistemas de evaluación continua y formativa; y (c) ecléctico (49\%), que se halla en una posición intermedia respecto a las actitudes. En el caso del profesorado innovador, hay una mayor predisposición para que el alumnado participe activamente en la evaluación, una menor predisposición por parte del profesorado ecléctico, pues suele utilizar innovaciones combinado con el examen final, mientras que el profesorado tradicional se muestra partidario de utilizar el examen final y/o combinado con un trabajo, como herramienta de evaluación y calificación.

Los estudios donde se relacionan las dos perspectivas, profesorado y alumnado, muestran habitualmente divergencias entre 
ellos. Los estudios revisados parecen indicar que el alumnado considera que es importante la evaluación formativa, el profesorado manifiesta la misma intención, pero aparecen divergencias, que se manifiestan en resistencias por ambas partes (Struyven, Dochy y Janssens, 2005). Por ejemplo, Gutiérrez, Pérez, Pérez y Palacios (2011) señalan que el profesorado es más abierto a la utilización de distintas formas de plantear la evaluación y entiende que no lo aprecia así el alumnado; mientras que el alumnado ve que la evaluación que utiliza su profesorado es "la de siempre”. Pero el profesorado también remarca que el alumnado tiene falta de compromiso, por ejemplo, cuando se trata de profundizar en los aprendizajes (Gijbels, Segers y Struyf, 2008; Lizzio, Wilson y Simons, 2002), incluso de la propia institución (Martínez, Castejón y Santos, 2012).

Según estos argumentos, parece necesario investigar a qué se debe que el alumnado no termine de comprometerse con un sistema de evaluación que ha demostrado beneficios para el aprendizaje. Por ello, el objetivo de este estudio es mostrar las divergencias del alumnado y del profesorado cuando se diseña y se ponen en práctica sistemas de evaluación formativa y sus resistencias a la hora de afrontar su aplicación, así como los inconvenientes que plantean sobre su uso.

\section{Método}

\section{Población}

La muestra es de 3013 estudiantes, pertenecientes a diferentes cursos y titulaciones, predominando las relacionadas con la Formación Inicial del Profesorado (FIP). Todos estos estudiantes han valorado los sistemas de evaluación formativa experimentados en las respectivas asignaturas durante el curso 2012-2013. La muestra es intencional, pues se eligió al profesorado y alumnado expresamente para el estudio. La muestra del alumnado pertenece a Magisterio (32 centros), Ciencias Actividad Física y Deporte (6 centros), y otras titulaciones (8 centros). En cuanto a los cursos, la población de estudiantes abarca desde primero a cuarto de Grado entre todas esas titulaciones. Contestaron el $100 \%$ de los alumnos que asisten regularmente a clase en dichos grupos.

Los 46 profesores de la muestra pertenecen a la Red Nacional de Evaluación Formativa en docencia universitaria. Ello implica el compromiso de aplicar sistemas de evaluación formativa en sus clases y presentar un autoinforme estructurado con los resultados de la experiencia de evaluación formativa implementada con sus alumnos universitarios a lo largo del curso 2012-2013. El alumnado es informado a comienzo de curso de que se va a aplicar un sistema de evaluación formativa en la asignatura, con lo que se les da una información con ejemplos prácticos para que entiendan y contrasten sus ideas sobre dicho concepto.

\section{Instrumentos}

Se utilizaron dos instrumentos para la recogida de datos: (a) el Auto informe anual de la red de Evaluación Formativa; y (b) el Cuestionario de los alumnos para la evaluación de la asignatura.

Auto informe anual de la red de Evaluación Formativa. Se trata de un autoinforme altamente estructurado sobre los resultados encontrados tras haber llevado a cabo un sistema de evaluación formativa y compartida en una asignatura de carácter cuatrimestral o anual. Se recogen datos durante el desarrollo de la asignatura y se redacta al finalizar la misma. Este instrumento ha sido validado $y$ aplicado en otras investigaciones publicadas, con lo que hay antecedentes que garantizan la aplicación de los informes (Buscà, Pintor, Martínez, y Peire, 2010; Manrique, Vallés y Gea, 2012; Vallés, Ureña y Ruiz, 2011). El auto-informe consta de las siguientes dimensiones: (1) Datos del contexto; (2) Relaciones del sistema de evaluación y aprendizaje; (3) Vías de aprendizaje y evaluación; (4) Aplicación de instrumentos de evaluación y orientación del aprendizaje; (5) Criterios éticos y carácter de las decisiones de la evaluación; (6) Desarrollo del sistema de evaluación a través de las TIC; 
(7) Ventajas que se reconocen en el sistema de evaluación aplicado; (8) Inconvenientes que se reconocen en el sistema de evaluación aplicado; (9) Rendimiento académico y valoración del mismo; (10) Carga de trabajo para alumnado y profesorado; y (11) Conclusiones.

En el apartado 7 el profesor responde a su grado de satisfacción con la implementación de sistemas de evaluación formativa en la experiencia y en el apartado 8 a percepciones sobre cómo lo habrán vivido sus alumnos, en ambos casos con una escala likert con cinco puntos de acuerdo: nada, poco, algo, bastante y mucho; además, en ambos apartados, vuelca los resultados de un cuestionario anónimo que sus alumnos han cumplimentado en relación a la misma experiencia, descrito a continuación.

Cuestionario sobre metodología y evaluación en formación inicial en Educación física (CMEFIEF). Se trata de un cuestionario anónimo para la evaluación de la asignatura por parte del alumnado. Fue validado por Castejón, Santos y Palacios (en prensa) con un Coeficiente Alpha de Cronbach para toda la escala de 0.84. Se siguió un proceso de validación de contenido, comprensión y fiabilidad del cuestionario en tres partes: (a) validez de contenido con expertos en la materia; (b) un estudio piloto con el fin de concretar el cuestionario; y (c) una aplicación del cuestionario a 892 estudiantes de Grado en formación inicial de distintas universidades. Para el cuestionario final se realizó un Análisis Factorial de Componentes Principales, donde se obtienen valores adecuados para el índice KMO de 0.807 como en el test de esfericidad de Barlett $(p>.00)$. Los índices obtenidos en la matriz de covarianzas, presentaron ajustes satisfactorios para el índice RMSEA $=0.078$, como en el GFI $=0.88$, así como en los restantes valores de ajuste del modelo.

Los estudiantes responden al grado de acuerdo con las afirmaciones allí reseñadas referidas a las ventajas e inconvenientes del sistema de evaluación, a través de una escala, también de tipo Likert, con cinco puntos de acuerdo: nada, poco, algo, bastante y mucho. La complementariedad de ambos instrumentos permite recoger la perspectiva del alumnado y poder contrastarla con la percepción del profesorado.

\section{Dimensiones y Variables}

Las dimensiones y variables se refieren a aspectos considerados como ventajas 0 inconvenientes de la aplicación de sistemas de evaluación formativa, obtenidos a partir de los informes de profesores de años anteriores, de las reuniones científicas y de la bibliografía especializada. Para este trabajo nos centramos en tres dimensiones (ver cada ítem en Tabla $1)$.

1. Inconvenientes_Alumnado. Grado de acuerdo de los alumnos con afirmaciones sobre la evaluación formativa entendidas como inconvenientes (14 ítems).

2. Inconvenientes_Profesorado. Opiniones del profesorado: (1) Auto percepción de la carga de trabajo que supone el sistema; (2) Percepción de las resistencias que presenta el alumnado ante la evaluación formativa (2 ítems).

3. Ventajas_Profesorado. Opiniones del profesorado: Auto percepción de la satisfacción con el sistema (1 ítem). 
Tabla 1. Dimensiones, ítems y variables que abordan los objetivos de nuestro estudio

\begin{tabular}{lll}
\hline \multicolumn{1}{c}{ Dimensión } & & \multicolumn{1}{c}{ Variables } \\
\hline & IA1 & Exige una asistencia obligatoria y activa \\
& IA2 & Tiene una dinámica de trabajo poco conocida, falta de hábito \\
& IA3 & Exige continuidad \\
& IA4 & Hay que comprenderlo previamente \\
& IA5 & Exige un mayor esfuerzo \\
& IA6 & Existe dificultad para trabajar en grupo \\
Inconvenientes & IA7 & Se puede acumular mucho trabajo al final \\
Alumno & IA8 & Existe una desproporción trabajo/créditos \\
& IA9 & El proceso de calificación es más complejo y, a veces, poco claro \\
& IA10 & Genera inseguridad e incertidumbre, dudas sobre qué hay que \\
& IA11 & Es injusto frente a otros procesos de evaluación \\
& IA12 & Las correcciones han sido poco claras \\
& IA13 & La valoración del trabajo es subjetiva \\
& IA14 & Exige participar en mi propia evaluación (autoevaluarse) \\
\hline Inconvenientes & IP1 & Supone una carga de trabajo excesiva para el profesor \\
Profesor & IP2 & El alumnado presenta fuertes resistencias frente al sistema de \\
& & evaluación continua y formativa \\
\hline Ventajas Profesor & VP1 & Satisfacción del profesor con el sistema de evaluación utilizado \\
\hline
\end{tabular}

\section{Procedimiento}

Un equipo de trabajo del Proyecto I+D+i sobre evaluación formativa en la formación inicial del profesorado diseñó un modelo de Auto-informe inicial y llevó a cabo un estudio piloto del mismo, efectuando posteriormente modificaciones y ajustes de sus características, hasta desembocar en el modelo que se aplicó en este estudio. El informe definitivo (explicado en el apartado de "Instrumentos") lo cumplimentó cada profesor tras concluir la asignatura sujeto de la experiencia de evaluación formativa. Las fechas de entrega para su uso por el resto de la Red de Evaluación Formativa son al final del primer o segundo semestre, dependiendo de cuándo termina la asignatura. Por su parte, los alumnos cumplimentaron el cuestionario anónimo antes señalado, al final de cada asignatura. Por término medio, cada estudiante tarda entre 30-40 minutos en completarlo. Los datos fueron enviados al coordinador, quien con el equipo de tratamiento de resultados volcaron la información de ambos instrumentos.

\section{Tratamiento de los datos}

Para el tratamiento de los datos se identificaron los cinco puntos de la escala ordinal, con valores numéricos; así el valor 0 se corresponde a la opción nada; el 1 a poco, el 2 a Algo, el 3 abastante y el 4 a mucho. Se tomó como referencia el procedimiento de análisis de Gutiérrez, Pérez y Pérez (2013) para un estudio semejante con profesores, alumnos y egresados y a Gutiérrez et al. (2011) para el tratamiento de las variables ordinales. Se realizaron estudios descriptivos y comparativos entre opiniones de profesores y de alumnos, en concreto: (1) Descriptivo tanto para las variables referidas a los profesores como a los alumnos (Medias y Desviación típica); (2) Comparativo entre ítems de profesorado (Prueba Rho de Spearman); (3) Comparativo entre variables de profesorado $\mathrm{y}$ de alumnos (Prueba de Kruskal Wallis). Se utilizaron los programas Excel_2007 y 
SPSS_v19, estableciéndose un nivel de significación de $p \leq 0.05$.

\section{Resultados}

Estudio descriptivo de las percepciones de los alumnos
En la Figura 1 presentamos los resultados sobre el grado de acuerdo de los estudiantes con catorce afirmaciones, consideradas como posibles inconvenientes respecto de la evaluación formativa en educación superior.

Figura 1. Grado de acuerdo o desacuerdo del alumnado con los posibles inconvenientes de los sistemas de evaluación formativa

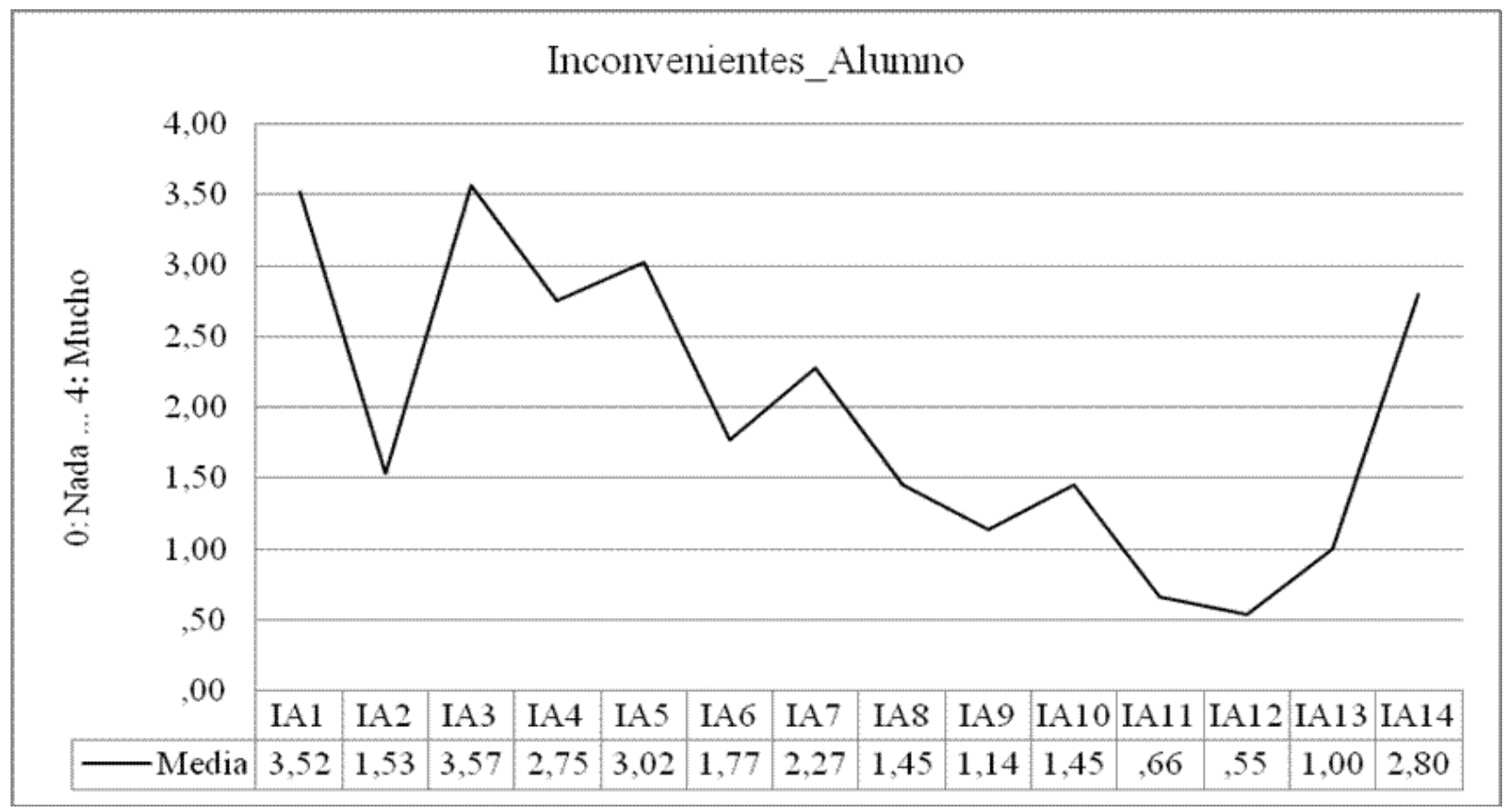

Como puede observarse, en ocho de los 14 ítems las puntuaciones se sitúan por debajo del valor 2 correspondiente a algo; tres ítems entre algo y bastante $\mathrm{y}$ otros tres ítems entre bastante ymucho. En más de la mitad de los ítems los valores otorgados por los alumnos a los inconvenientes son bajos y tan solo en tres de los casos, poco más del $20 \%$, les conceden valores altos, siendo la media global de $M=$ 1.87, por debajo de algo. Para situar mejor la comprensión de los datos, los hemos organizado en tres apartados: (a) aquéllos que se refieren a los aspectos de procedimiento y organización; (b) los que se centran en exigencias del sistema de evaluación; y (c) los que conciernen a la calificación.
Procedimiento y organización (aplicación). En la Tabla 2 presentamos los datos referidos a los posibles inconvenientes en la aplicación (procedimiento, organización...) de los sistemas de evaluación formativa en educación superior (ítems 2, 4, 6, 7, 8 y 10). En ellos encontramos cuatro valores entre poco y algo; los dos más próximos a poco hacen referencia a la desproporción entre trabajo y créditos de la asignatura $(M=1.45)$ y a si se genera inseguridad e incertidumbre $(M=1.45)$. Los dos más próximos a algo se refieren a si el sistema de evaluación tiene una dinámica de trabajo poco conocida $(M=1.53)$ y a la dificultad para trabajar en grupo $(M=1.77)$. 
Tabla 2. Posibles inconvenientes en la aplicación de los sistemas de evaluación formativa en educación superior

\begin{tabular}{lllc}
\hline Ítems & Variable & Media & Desv.Típ. \\
\hline IA2 & Tiene una dinámica de trabajo poco conocida, falta de hábito & 1.53 & 1.162 \\
IA4 & Hay que comprenderlo previamente & 2.75 & 0.651 \\
IA6 & Existe dificultad para trabajar en grupo & 1.77 & 1.008 \\
IA7 & Se puede acumular mucho trabajo al final & 2.27 & 1.042 \\
IA8 & Existe una desproporción trabajo/créditos & 1.45 & 0.926 \\
IA10 & Genera inseguridad e incertidumbre. Dudas sobre qué hay que realizar & 1.45 & 0.901 \\
\hline
\end{tabular}

Entre los ítems que muestran un cierto grado de acuerdo con estos posibles inconvenientes encontramos el ítem IA7, que hace referencia a que se puede acumular mucho trabajo al final $(M=2.27)$. Por otro lado, los estudiantes afirman estar bastante de acuerdo con que es importante la comprensión previa del sistema de evaluación formativa (IA4, $M=2.75)$.

Grado de exigencia. La Figura 1 muestra que los valores más altos que otorgan los alumnos a todos los inconvenientes, son los cuatro cuyo enunciado incluye una exigencia (ítems 1, 3, 5 y 14; en la Tabla 3). El mayor grado de acuerdo hace referencia a que los sistemas de evaluación formativa exigen continuidad $(M=3.57)$, una asistencia obligatoria y activa $(M=3.52)$, un mayor esfuerzo $(M=3.02)$ y a participar en su propia evaluación, es decir a autoevaluarse $(M=$ 2.809).

Tabla 3. Grado de acuerdo sobre las exigencias que implican los sistemas de evaluación formativa

\begin{tabular}{clcc}
\hline Ítems & Variable & Media & Desv.Típ. \\
\hline IA1 & Exige una asistencia obligatoria y activa & 3.52 & .762 \\
IA3 & Exige continuidad & 3.57 & .587 \\
IA5 & Exige un mayor esfuerzo & 3.02 & .590 \\
IA14 & Exige participar en mi propia evaluación & 2.80 & 1.112 \\
\hline
\end{tabular}

Calificación. La tabla 4 muestra cómo el alumnado considera muy poco importantes los posibles inconvenientes que pueda tener la calificación en las asignaturas que utilizan sistemas de evaluación formativa (ítems 9, 11, 12 y 13). Se obtienen valores muy bajos en todos ellos, especialmente en el que hace referencia a la posible injusticia frente a otros procesos de evaluación $(M=.66)$ y en el relativo a la falta de claridad en las correcciones $(M=.55)$.

Tabla 4. Posibles inconvenientes en relación al proceso de calificación.

\begin{tabular}{llcc}
\hline Ítems & Variable & Media & Desv.Típ. \\
\hline IA9 & El proceso de calificación es más complejo y, a veces, poco claro & 1.14 & .930 \\
IA11 & Es injusto frente a otros procesos de evaluación & 0.66 & .861 \\
IA12 & Las correcciones han sido poco claras & 0.55 & .820 \\
IA13 & La valoración del trabajo es subjetiva & 1 & .964 \\
\hline
\end{tabular}

Estudio descriptivo de las opiniones del profesorado 
En cuanto a las tres variables referidas al profesorado, en la Tabla 5 puede observarse cómo el profesorado que desarrolla sistemas de evaluación formativa en sus asignaturas, muestra un elevado grado de satisfacción $(M=$ 3.31)

En relación a los posibles inconvenientes que este colectivo encuentra, los datos nos indican que el profesorado considera de forma mayoritaria que la evaluación formativa le supone una alta carga de trabajo $(M=3.02)$; por el contrario, no considera que el alumnado presente fuertes resistencias al sistema de evaluación continua y formativa $(M=1.18)$.

Tabla 5. Opiniones del profesorado: satisfacción y posibles inconvenientes.

\begin{tabular}{llll}
\hline Ítems & Variable & Media & Desv.Típ. \\
\hline VP1 & Satisfacción del profesor con el sistema de evaluación utilizado & 3.31 & .569 \\
IP1 & Supone una carga de trabajo excesiva para el profesor & 3.02 & .79018 \\
IP2 & $\begin{array}{l}\text { El alumnado presenta fuertes resistencias frente al sistema de evaluación } \\
\text { continua y formativa }\end{array}$ & 1.18 & .95451 \\
\hline
\end{tabular}

Comparación entre opiniones del profesor

Pretendemos conocer el grado de satisfacción del profesorado con el sistema de evaluación formativa utilizado, en relación a si la carga de trabajo que le supone es excesiva (VP1 vs. IP1).

Tabla 6. Comparación de satisfacción y carga de trabajo del profesor

\begin{tabular}{ccccc}
\hline $\begin{array}{c}\text { Comparación VP1 } \\
\text { vs. }\end{array}$ & $\begin{array}{c}\text { Media } \\
\text { VP1 }\end{array}$ & $\begin{array}{c}\text { Media } \\
\text { IP1 }\end{array}$ & Coeficiente de correlación & Sig. (bilateral) \\
\cline { 2 - 5 } IP1_Rho de Spearman & 3.31 & 3.02 & -0.067 & 0.688 \\
\hline
\end{tabular}

La media de las dos variables es alta (como se observa en la Tabla 6), puesto que supera en ambos casos el valor 3, bastante. El valor del coeficiente de correlación, próximo a cero y su signo negativo, nos indican una correlación inversa muy débil, pero no hay significación al darse un $p=.688$, por tanto dichas variables no están correlacionadas y los profesores mantienen pares de opiniones heterogéneas.

Comparación profesor-alumnos

Se pretende comparar la percepción que el profesorado tenía sobre las resistencias de los alumnos ante la evaluación formativa, con la opinión de los propios alumnos al respecto y qué aspectos concretos pudieran motivar esas posibles resistencias.

Según el profesorado, los estudiantes iban a manifestar pocos desacuerdos con el sistema de evaluación formativa pues otorgaron a esa variable (IP2) una media de $M=1.18$, mientras que la media global de las opiniones de los alumnos fue mayor $(M=2)$. Para profundizar en esta cuestión, comparamos la variable profesor (IP2) con cada variable de los alumnos (IA1, IA2..., IA14), mediante la prueba de Kruskal-Wallis y los resultados fueron los de la Tabla 7. 
Tabla 7. Comparación entre percepciones de profesores y opiniones de los alumnos sobre resistencias a la evaluación formativa

\begin{tabular}{ccccc}
\hline & & & \multicolumn{2}{c}{$\begin{array}{c}\text { Resumen de la prueba de hipótesis } \\
\text { EruskalWallis_significancia .05 }\end{array}$} \\
\hline IP2 vs. IA1 & 1.18 & 3.52 & .055 & aceptar \\
IP2 vs. IA2 & 1.18 & 1.53 & .945 & aceptar \\
IP2 vs. IA3 & 1.18 & 3.57 & .370 & aceptar \\
IP2 vs. IA4 & 1.18 & 2.75 & .231 & aceptar \\
IP2 vs. IA5 & 1.18 & 3.02 & .065 & aceptar \\
IP2 vs. IA6 & 1.18 & 1.77 & .356 & aceptar \\
IP2 vs. IA7 & 1.18 & 2.27 & $.031 *$ & rechazar \\
IP2 vs. IA8 & 1.18 & 1.45 & .161 & aceptar \\
IP2 vs. IA9 & 1.18 & 1.14 & .461 & aceptar \\
IP2 vs. IA10 & 1.18 & 1.45 & .779 & aceptar \\
IP2 vs. IA11 & 1.18 & 0.66 & $.019 *$ & rechazar \\
IP2 vs. IA12 & 1.18 & 0.55 & $.018 *$ & rechazar \\
IP2 vs. IA13 & 1.18 & 1.00 & .061 & aceptar \\
IP2 vs. IA14 & 1.18 & 2.80 & .113 & aceptar \\
\hline
\end{tabular}

Vemos que en 11 de las 14 comparaciones, el valor de significación supera el $p=.05$ por lo que en esos once casos aceptamos la hipótesis de igualdad, es decir, entre las percepciones del profesorado y la opinión del alumnado no hubieron discrepancias significativas; en otras tres, marcadas con asterisco, IA7 (Se puede acumular mucho trabajo al final; $M=2.27$ ); IA11 (Es injusto frente a otros procesos de evaluación; $M=$ .66), IA12 (Las correcciones han sido poco claras; $M=.55$ ), sí hubieron discrepancias entre alumnos y profesores.

Para saber en qué sentido fueron esas discrepancias, comparamos las medias de alumnos y profesorado y observamos que los alumnos daban más importancia que sus profesores al hecho de que se puedan acumular tareas al final del proceso (IP2 vs IA7). Por el contrario los alumnos consideraron menos importante que sus profesores la claridad o injusticia en la calificación (IP2 vs. IA11 e IA12).

\section{Discusión}

\section{Percepciones de los alumnos}

En el estudio de las percepciones de los alumnos sobre posibles inconvenientes de la evaluación formativa, algunos autores destacaban que el alumnado mostraba ciertas resistencias a la hora de participar en este tipo de sistemas de evaluación (Castejón et al., 2011; Julián et al., 2010; López-Pastor, 2008; López-Pastor et al., 2011; Pérez et al., 2008; Capllonch et al., 2009); en nuestro estudio los alumnos no presentaron altas resistencias en general, pero sí lo hicieron en determinados aspectos concretos, en la línea de otros trabajos que señalaban que esas resistencias se daban en diferentes momentos del proceso, desde el diseño y puesta en práctica (LópezPastor, 2009; Sadler, 2005), hasta la retroalimentación (Carless, 2006).

Procedimiento y organización. En cuanto a cuestiones de procedimiento y organización, cuatro aspectos, que a priori podrían parecer inconvenientes, fueron poco destacables para los alumnos, es decir, no encontraron 
demasiados problemas para trabajar en grupo, se les generó poca incertidumbre o dudas sobre lo que tenían que hacer y además, no consideraron que existiera una desproporción entre los créditos y la cantidad de trabajo, coincidiendo con otros estudios (Julián et al., 2010; López et al., 2011; Romero-Martín et al., 2014), que parecen demostrar que la utilización de sistemas de evaluación formativa no genera una sobrecarga de trabajo real para el alumnado, sino que está dentro de los parámetros de horas de trabajo que suponen los créditos ECTS en cada asignatura.

El alumnado no consideró un inconveniente destacable la falta de costumbre en este tipo de sistemas de evaluación, lo cual guarda relación con los estudios de Gutiérrez et al. (2011); Palacios y López-Pastor (2013) y Palacios, López-Pastor y Barba (2013), sobre el incremento paulatino del porcentaje de profesorado universitario que desarrolla sistemas de evaluación formativa y continua en la universidad española.

En lo relativo a la distribución del trabajo a lo largo del curso, los resultados indicaron que al menos una pequeña parte del profesorado que utilizó sistemas de evaluación formativa no repartía correctamente las tareas a lo largo de todo el cuatrimestre, generando una mayor acumulación de trabajo al final del periodo lectivo, precisamente cuando los estudiantes tienen que dedicar mayor tiempo a preparar las asignaturas que mantienen los tradicionales exámenes finales. Esto coincide con los resultados de Hamodi y López (2012), que recogieron $\mathrm{y}$ estudiaron las valoraciones negativas del alumnado sobre esta costumbre en la universidad española, así como los de Julián et al. (2010), que demostraron en su estudio que el hecho de no fijar plazos para la entrega de las diferentes actividades y evidencias a lo largo de todo el cuatrimestre generaba una acumulación excesiva de trabajo al final del mismo y, ligado a ello, una mayor tasa de fracaso y/o abandono. Todo ello refuerza la idea de que en los sistemas de evaluación formativa es fundamental establecer plazos de realización y entrega de la mayoría de las actividades de aprendizaje en los tres primeros meses del cuatrimestre, para evitar este problema de acumulación excesiva de trabajo al final del cuatrimestre.

Nuestros estudiantes consideraron importante comprender el sistema previamente, lo que coincide con Castejón et al. (2011), quienes daban mucha importancia a dejar claro un buen procedimiento de actuación, lo cual evitaría originar una peligrosa confianza en la seguridad de éxito tal como indicaban Buscà et al. (2010).

Por tanto, no podemos decir que los factores referidos a la organización de los sistemas de evaluación constituyeran inconvenientes insalvables, aunque varios ítems aportaron datos importantes para conocer por donde poder ir mejorando la implementación de la evaluación formativa.

Grado de exigencia. Los estudiantes afirmaron que el sistema de evaluación formativa exigía un mayor esfuerzo que otros tipos de evaluación; que implicaba continuidad en el trabajo y en la asistencia y que esta asistencia debía ser activa; por lo que cabe suponer que el grado de implicación personal del alumno no pudo ser superficial. Esta implicación puede facilitar la puesta en práctica de procesos de auto-regulación (Black y Wiliam, 2009 y Yorke, 2003), teniendo un impacto positivo sobre la participación, sobre la disminución de la tasa de abandonos entre el alumnado y ejerciendo efectos positivos sobre el aprendizaje (López-Pastor, Pintor, Muros y Webb, 2013) y el rendimiento académico (Biggs, 2005; Boud y Falchikov, 2007; Bowden y Marton, 2012; Brown y Glasner, 2007; Cano, 2012; Carrillo et al., 2009; Castejón et al., 2011; Knight, 2005; Marton y Säljö, 1976; Romero-Martín et al., 2014).

Calificación. Los resultados encontrados desmontan algunos de los prejuicios existentes sobre este tipo de sistemas de evaluación. A pesar de la cantidad y diversidad de actividades de aprendizaje que suelen tenerse en cuenta en los sistemas de evaluación formativa, los alumnos de nuestro estudio no 
consideraron que el proceso de calificación hubiera sido más complejo o poco claro. Es más, señalaron que la valoración del trabajo no había sido tan subjetiva y destacaron, por positivas, las valoraciones sobre la justicia del proceso en general y la claridad de las correcciones, lo que podría interpretarse como que el profesorado diseñó y aplicó técnicas didácticas eficaces en la línea de las recomendaciones de Castejón et al. (2011) sobre la implementación de la evaluación formativa.

Los resultados nos parecen especialmente interesantes dada la conocida preocupación por la calificación; por un lado de los estudiantes $y$, por otro, del docente, responsable de que las calificaciones sean reflejo veraz del proceso de aprendizaje del alumnado. A tal fin, varios estudios sobre evaluación formativa en la formación inicial del profesorado encontraron mejoras considerables en las calificaciones del alumnado (Castejón et al., 2011; López-Pastor, 2008; López-Pastor et al., 2011), lo que en nuestro estudio pudo haber influido en la positiva percepción de los estudiantes en relación a la calificación.

\section{Opiniones del profesorado}

El grado de satisfacción del profesorado de nuestro estudio con la evaluación formativa fue alto; no hay que olvidar que se trataba de profesores que voluntariamente habían realizado una experiencia de evaluación formativa, por tanto que respondían al perfil de profesor innovador, de acuerdo con Palacios y López-Pastor (2013) y Palacios et al. (2013), quienes lo describían como profesorado con actitudes positivas hacia la evaluación continua y formativa, es decir que habían superado suficientemente las resistencias a las que se referían Pérez et al. (2008) y Capllonch et al. (2009); por tanto, las valoraciones altas en satisfacción confirmaron lo esperado.

Además el profesorado consideró que la carga de trabajo fue elevada, en la línea de otros estudios que situaban como principal inconveniente de la evaluación formativa el incremento del tiempo y la dedicación del profesorado (Bennet, 2011; Gibbs y Simpson, 2004-05).

La comparación entre esas dos variables mostraba una ligera tendencia a que el profesorado estuviera satisfecho a pesar de tener una carga excesiva, posiblemente al ser consciente de que ésta se encontraba dentro de los estándares de lo que suponen para el profesorado universitario los créditos ECTS de las distintas asignaturas, en la línea de los estudios de Julián et al. (2010) y López-Pastor et al. (2013), que con datos reales de carga de trabajo concluían que son perfectamente viables. Sin embargo la correlación no fue significativa, por lo que no se ha podido confirmar esos términos.

\section{Comparativa percepciones del profesor- opinión de los alumnos}

A pesar de que determinada literatura señalaba que el profesorado percibía resistencias a la evaluación formativa por parte del alumnado (Capllonch et al., 2009; Pérez et al., 2008; Struyven et al., 2005), los profesores participantes en nuestro estudio consideraron que éstas serían escasas, posiblemente porque su percepción del día a día les había dado indicaciones de la buena aceptación en general que luego demostró el alumnado. Los resultados nos confirmaron que, en un alto porcentaje, las opiniones de los alumnos coincidieron con las percepciones de los profesores.

De los tres casos en los que se dieron discrepancias, en dos los alumnos puntuaron aun más bajo esos inconvenientes de lo esperado por el profesorado, concretamente en "Es injusto frente a otros procesos de evaluación” y "Las correcciones han sido poco claras". Hay que reconocer que es un dato inesperado ya que la calificación suele generar desacuerdos entre profesores y alumnos, pero si la evaluación formativa pretende que los estudiantes sean aprendices independientes y críticos (Torrance, 2012), debemos entender que sus opiniones se han hecho desde esos presupuestos. 
En el tercer caso en el que hubo discrepancia entre profesorado y alumnado referido a si se puede acumular mucho trabajo al final, las puntuaciones de los alumnos fueron más altas que las del profesorado. Este dato es muy relevante si tenemos en cuenta que uno de los valores fundamentales del proceso de evaluación formativa es la continuidad, en la medida en que ésta permite la aplicación de retroalimentación constante para reconducir el aprendizaje y así superar la brecha entre lo que el alumno sabe y lo que debe saber (Taras, 2009; Torrance, 2012); en definitiva, para permitir un adecuado uso de la información por parte del alumno para mejorar su aprendizaje, lo que a su vez, repercutirá en su motivación y en su rendimiento, como se manifiesta en los estudios que han investigado al respecto (por ejemplo, Burke, 2009; Carless, 2006; Carless et al., 2010).

Sin duda estos resultados permiten al profesorado ajustar sus percepciones e indican por dónde podrán mejorarse los sistemas de evaluación formativa aplicados.

\section{Conclusiones}

La investigación científica sobre el impacto de la evaluación formativa parece ser aún insuficiente. El cúmulo de estudios que han aportado información relevante al respecto ha sido comparando las ventajas o bien, aportando información que corroboren lo que otros estudios han notificado. En esta línea marcada por Dunn y Mulvenon (2009), el presente estudio ha aportado datos sobre una experiencia concreta, lo que supone contribuir con información relevante para que el profesorado universitario pueda adoptar la evaluación formativa sabiendo que tiene que actuar de forma adecuada y con argumentos suficientes. De acuerdo al objetivo del presente estudio, se ha corroborado que existen divergencias entre las opiniones de profesorado y alumnado respecto a la evaluación formativa. El alumnado suele mostrar ciertas resistencias sobre la evaluación formativa aunque en nuestro estudio no han sido tan elevadas como cabría esperar las relativas a la puesta en práctica y la excesiva carga de trabajo, debido posiblemente a que el alumnado ha experimentado este tipo de evaluación en más cursos y asume esa práctica con naturalidad. Sin embargo, los resultados nos indican que sería conveniente que el profesorado planificara con mayor precisión el reparto de la carga de trabajo a lo largo del cuatrimestre, para evitar la sobresaturación de las últimas semanas, cuando se suman las exigencias de las asignaturas de evaluación continua con las de asignaturas que siguen apostando por una evaluación fundamentalmente final. La percepción de carga de trabajo parece mejorar cuando se fijan plazos que ayudan al alumnado a ajustarse mejor a la evaluación formativa, evitando la tasa de abandono y fracaso que tiende a generarse cuando los plazos son demasiado flexibles.

Otra conclusión que confirma el objetivo del estudio es que implicar al alumnado en el diseño y proceso de evaluación ayuda a mejorar la predisposición hacia la misma, aunque lo consideren un sistema exigente en asistencia y dedicación; no parece ser que esto les haga tener una valoración negativa del conjunto. Efectivamente, la experimentación por el alumnado de la evaluación formativa permite que su auto-regulación para la evaluación y el aprendizaje mejoren. Los estudiantes también valoran positivamente la relación entre procesos de evaluación formativa y calificación; no consideran que sean procesos más injustos ni plantean problemas en relación a esta temática. Parecen valorarse positivamente las relaciones entre el grado de implicación, la diversidad de actividades de aprendizaje que se utilizan y la calificación obtenida.

En cuanto al profesorado, considera que esos sistemas requieren una alta dedicación pero también suponen una elevada satisfacción por las ventajas su supone para el alumnado. Parece ser que dicha dedicación se encuentra dentro de las exigencias de los créditos ECTS, pero los datos no son tan claros como en otros estudios. 
La comparativa entre profesorado y alumnado muestra que hay un alto grado de coincidencia entre los dos grupos de nuestro estudio en torno al $80 \%$ de los ítems, mientras que solo aparecen diferencias en unos pocos (el 20\%), del tipo: es injusto frente a otros procesos de evaluación, las correcciones han sido poco claras y se puede acumular mucho trabajo al final. Son divergencias fácilmente solucionables, según se ha ido expresando a lo largo del estudio: con mejor planificación por parte del profesorado y con más implicación por parte del alumnado.

Este estudio tiene limitaciones, particularmente las derivadas de que el profesorado que participa en este estudio lleva años aplicando la evaluación formativa $y$ debería contrastarse con profesorado que no sigue esta forma de aplicar la evaluación, incluso con profesorado que tuviera diferentes grados de experiencia en llevar a cabo la evaluación formativa. Lo mismo se podría tener en cuenta respecto del alumnado, sus aportaciones y percepciones cuando tienen asignaturas con evaluación formativa y cuando no la tienen. Algunos aspectos a explorar que parecen tener especial relevancia son las ayudas en la planificación de las exigencias y evidencias que tienen que presentar los estudiantes y el feedback que debe utilizar el profesorado para que los procesos de autoregulación mejoren más de lo que habitualmente se consigue.

\section{Referencias}

Bennet, R. E. (2011). Formative assessment: A critical review. Assessment in Education: Principles, Policy \& Practice, 18, 5-25. DOI:10.1080/0969594X.2010.513678

Biggs, J. (2005). Calidad del aprendizaje universitario. Madrid: Narcea.

Black, P. \& Wiliam, D. (2009). Developing the theory of formative assessment. Educational Assessment, Evaluation and Accountability, 21, 5-31. DOI: 10.1007/s11092-008-9068-5

Boud, D. \& Falchikov, N. (2007). Rethinking Assessment in Higher Education. Learning for the long term. Londres: Routledge

Boussada, H. \& De Ketele, J-M. (2008). L'évaluation de la qualité de la formation et du système d'évaluation universitaire: Le point de vue des diplômés. Avaliação, 13(1), 39-61. DOI:10.1590/S1414-40772008000100003

Bowden, J. \& Marton, F. (2012). La Universidad. Un espacio para el aprendizaje. Madrid: Narcea.

Brown, S. \& Glasner, A. (Eds.) (2007). Evaluar la Universidad. Problemas y nuevos enfoques ( $2^{\mathrm{a}}$ ed.). Madrid: Narcea.

Burke, D. (2009). Strategies for using feedback students bring to higher education. Assessment \& Evaluation in Higher Education, 34(1), 41-50. DOI: 10.1080/02602930801895711

Buscà, F., Pintor, P., Martínez-Mínguez, L. \& Peire, T. (2010). Sistemas y procedimientos de Evaluación Formativa en docencia universitaria: Resultados de 34 casos aplicados durante el curso académico 2007-2008. Estudios sobre Educación, 18, 255-276. Recuperado de http://dspace.unav.es/dspace/bitstream/10171/982 9/2/ESE 18 11.pdf

Cano, E. (Ed.) (2012). Aprobar o aprender. Estrategias de evaluación en la sociedad red. Barcelona: Laboratori de Mitjans Interactius.

Capllonch, M., Ureña, N., Ruiz, E., González, N., Pérez, A., López, V. M \& Tabernero, B. (2009). Guía de problemas y soluciones para el desarrollo de sistemas de evaluación formativa en la universidad. En V. M. López Pastor, (coord.), La Evaluación Formativa y Compartida en Docencia Universitaria: propuestas, técnicas, instrumentos y experiencias (pp. 217252).Madrid: Narcea.

Carless, D. (2006). Differing perceptions in the feedback process. Studies in Higher Education, 31(2), 219-33. DOI: $\underline{10.1080 / 03075070600572132}$

Carless, D., Salter, D., Yang, M. \& Lam, J. (2010).Developing sustainable feedback practices. Studies in Higher Education, 36(4), 395-407. DOI: 10.1080/03075071003642449

Carrillo, M.T., Baillès, E., Caseras, X., Martínez, A., Ortet, G. \& Pérez, J. (2009). Formative assessment and academic achievement in pregraduate students of health sciences. Advances in Health Sciences Education, 14, 61-67. DOI: 10.1007/s10459-007-9086-y

Castejón, F. J., López-Pastor, M. L.; Julián, J. \& Zaragoza, J. (2011). Evaluación formativa y rendimiento académico en la Formación inicial del profesorado de Educación Física. Revista Internacional de Medicina y Ciencias de la Actividad Física y el Deporte, 11(42), 328-346. 
Recuperado

de

http://cdeporte.rediris.es/revista/revista42/arteval uacion163.htm

Castejón, F. J., Santos, M.L. \& Palacios, A. (En prensa). Cuestionario sobre metodología y evaluación en formación inicial en educación física. Revista Internacional de Medicina y Ciencias de la Actividad Física y el Deporte. Recuperado de http://cdeporte.rediris.es/revista/inpress/artescala 566.pdf.

De la Calle, M.J. (2004). El reto de ser profesor en el contexto de la convergencia europea. La formación pedagógica como necesidad. Revista Universitaria de Formación del Profesorado, 18(3), 251-258. Recuperado de http://www.redalyc.org/articulo.oa?id=27418316

Dunn, K. E. \& Mulvenon, S. W. (2009). A critical review of research on formative assessment: The limited scientific evidence of the impact of formative assessment in education. Practical Assessment, Research \& Evaluation, 14(7), 1-11.

Falchikov, N. y Goldfinch, J. (2000). Student peer assessment in higher education: A meta-analysis comparing peer and teacher marks. Review of Educational Research, 70(3), 287-322. DOI: 10.3102/00346543070003287

Fallows, S. \& Chandramohan, B. (2001). Multiple Approaches to Assessment: reflections on use of tutor, peer and self-assessment. Teaching in Higher Education, 6(2), 229-246. DOI: $\underline{10.1080 / 13562510120045212}$

Fernández, M. (1989). Así enseña nuestra universidad: Hacia la construcción crítica de una Didáctica universitaria. Madrid: UCM.

Gibbs, G. \& Simpson, C. (2004-05). Conditions under which assessment supports students' learning. Learning and Teaching in Higher Education, 1, 3-31.

Gijbels, D. \& Dochy, F. (2006). Students' assessment preferences and approaches to learning: can formative assessment make a difference? Educational Studies, 32(4), 399-409. DOI: $10.1080 / 03055690600850354$

Gijbels, D., Segers, M. \& Struyf, E. (2008). Constructivist learning environments and the (im)possibility to change students' perceptions of assessment demands and approaches to learning.Instructional Science, 36, 431-443. DOI: 10.1007/s11251-008-9064-7

Gutiérrez-García, C., Pérez-Pueyo, A. \& PérezGutiérrez, M. (2013). Percepciones de profesores, alumnos y egresados sobre los sistemas de evaluación en estudios universitarios de formación del profesorado de Educación Física. Ágora para la Educación Física y el Deporte, 15(2), 130-151. Recuperado de http://agorarevista.blogs.uva.es/files/2013/11/agora_15_2d_g utierrez_et_al.pdf

Gutiérrez, C., Pérez, Á., Pérez, M. \& Palacios, A. (2011). Percepciones de profesores y alumnos sobre la enseñanza, evaluación y desarrollo de competencias en estudios universitarios de formación de profesorado. Cultura y Educación, 23(4), 499-514. DOI: $\underline{10.1174 / 113564011798392451}$

Hamodi, C. \& López-Pastor, A. T. (2012). La evaluación formativa y compartida en la Formación Inicialdel Profesorado desde la perspectiva del alumnado y de los egresados. Psychology, Society, \& Education, 4(1), 103-116.

Knight, P. T. (2005). El profesorado de Educación Superior. Madrid: Narcea.

Krasner, S., Wimmers, P. F., Relan, A. \& Drake, T. A. (2006). Differential Effects of Two Types of Formative Assessment in Predicting Performance of First-year Medical Students. Advances in Health Sciences Education, 11, 155171. DOI: $10.1007 / \mathrm{s} 10459-005-5290-9$

Julián, J., Zaragoza, J., Castejón, F. J. \& LópezPastor, V. M. (2010). Carga de trabajo en diferentes asignaturas que experimentan el sistema ECTS. Revista Internacional de Medicina y Ciencias de la Actividad Física y el Deporte, 10(38), 218-233. Recuperado de http://cdeporte.rediris.es/revista/revista38/artcarg a151.htm

Lizzio, A., Wilson, K. \& Simons, R. (2002). University Students' Perceptions of the Learning Environment and Academic Outcomes: implications for theory and practice. Studies in HigherEducation, 27(1), 27-52. DOI: 10.1080/0307507012009935 9

López-Pastor, V. M. (2008). Desarrollando sistemas de evaluación formativa y compartida en la docencia universitaria. Análisis de resultados de su puesta en práctica en la formación inicial del profesorado. European Journal of Teacher Education, 31(3), 293-311. DOI: $10.1080 / 02619760802208452$

López-Pastor, V. M. (Ed.) (2009). Evaluación Formativa y Compartida en Educación Superior. Madrid: Narcea. 
López-Pastor, V. M., Manrique, J.C. \& Vallés, C. (2011). Evaluación y calificación en los nuevos estudios de Grado. Especial incidencia en la Formación Inicial del Profesorado. Revista Electrónica Interuniversitaria de Formación del Profesorado, 39(14.4), 1-20. Recuperado de http://www.aufop.com/aufop/revistas/arta/digital/ $165 / 1675$

López-Pastor, V. M., Pintor, P., Muros, B. \& Webb, G. (2013). Formative assessment strategies and their effects on student performance and on student and tutor workload: The results of research projects undertaken in preparation for greater convergence os universities in Spain within the European Higher Education Area (EHEA). Journal of Further and Higher Education, 37(2), 163-180. DOI: 101080/0309877X.2011.644780

Manrique, J. C., Vallés, C. \& Gea, J. M. (2012). Resultados generales de la puesta en práctica de 29 casos sobre el desarrollo de sistemas de Evaluación formativa en docencia universitaria.Psychology, Society \& Education, 4(1), 83-98. Recuperado de http://www.psye.org/articulos/vista\%20Santos\%2 0Pastor.pdf

Martínez, L. F., Castejón, F. J. \& Santos, M. L. (2012). Diferentes percepciones sobre evaluación formativa entre profesorado y alumnado en formación inicialen educación física. Revista Electrónica Interuniversitaria de Formación del Profesorado, 15(4), 57-67. Recuperado de http://www.aufop.com/aufop/revistas/arta/digital/ $\underline{171 / 1747}$

Marton, F. \& Säljö, R. (1976). On Qualitative Differences in Learning-2: Outcome as a function of the learner's conception of the task. British Journal of Educational Psychology, 46, 115127.DOI: 10.1111/j.2044-8279.1976.tb02304.x

Nicol, D. J. \& Macfarlane-Dick, D. (2006). Formative assessment and self-regulated learning: A model and seven principles of good feedback practice. Studies in HigherEducation, 31(2), 199-218. $\underline{10.1080 / 03075070600572090}$

Palacios, A. \& López-Pastor, V. M. (2013). Haz lo que yo digo pero no lo que yo hago: sistemas de evaluación del alumnado en la formación inicial del profesorado. Revista de Educación, 361, 279305. DOI: 10.4438/1988-592X-RE-2011-361$\underline{143}$

Palacios, A., López-Pastor, V. M. \& Barba, J. J. (2013). Tipologías de profesorado universitario en función de la evaluación aplicada a los futuros docentes. Estudios sobre educación, 24, 173-195. Recuperado http://dspace.unav.es/dspace/bitstream/10171/295 69/2/PALACIOS.pdf

Pérez, A., Tabernero, B., López, V. M., Ureña, N., Ruiz, E., Capllonch, M. \& Castejón, J. (2008). Evaluación formativa y compartida en la docencia universitaria: La concreción de cuestiones/clave para su aplicación en el camino hacia el Espacio Europeo de Educación Superior (EEES). Revista de Educación, 347, 435-451. Recuperado de http://www.mecd.gob.es/dctm/revista-deeducacion/articulosre347/re34720.pdf?documentI $\mathrm{d}=0901 \mathrm{e} 72 \mathrm{~b} 8123677 \mathrm{c}$

Romero-Martín, R., Fraile-Aranda, A., LópezPastor, V. M. \& Castejón-Oliva, F. J. (2014). Relación entre sistemas de evaluación formativa, rendimiento académico y carga de trabajo del profesor y del alumno en la docencia universitaria. Infancia y Aprendizaje, 37(2), 310341. DOI: $10.1080 / 02103702.2014 .918818$

Rué, J. (2009). El aprendizaje autónomo en la Educación Superior. Madrid: Narcea.

Sadler, E. R. (2005). Interpretations of criteriabased assessment and grading in higher education. Assessment \& Evaluation in Higher Education, 30(2), 175-194. DOI: 10.1080/0260293042000264262

Santos, M. L., Martínez, L. F. \& López-Pastor, V. M. (Eds.) (2009). La innovación docente en el EEES. Experiencias de evaluación formativa y compartida en la formación inicial del profesorado. Universidad de Almería.

Struyven, K., Dochy, F. \& Janssens, S. (2005). Students' perceptions about evaluation and assessment in higher education: a review. Assessment \& Evaluation in Higher Education, 30(4), 325-341. DOI: 10.1080/02602930500099102

Taras, M. (2009). Summative assessment: The missing link for formative assessment. Journal of Further and Higher Education, 33, 57-69. DOI: $\underline{10.1080 / 03098770802638671}$

Tejedor, F. J. (Coord.) (1998). Los alumnos de la Universidad de Salamanca. Universidad de Salamanca.

Tonucci, F. (2010). Apuntes sobre el manifiesto. Educar(nos), 49, 9-11.

Torrance, H. (2012). Formative assessment at the crossroads: conformative, deformative and 
transformative assessment. Oxford Review of Education, 38(3), 323-342. DOI: $\underline{10.1080 / 03054985.2012 .689693}$

Trillo, F. (2005). La evaluación de los estudiantes universitarios. El caso de la Universidad de Santiago de Compostela. Universidad de Santiago de Compostela.

Vallés, C., Ureña, N. \& Ruiz, E. (2011). La evaluación formativa en docencia universitaria.
Resultados globales de 41 estudios de caso. REDU, 9(1), 135-158.

Yorke, M. (2003). Formative assessment in higher education: Moves towards theory and the enhancement of pedagogic practice. Higher Education, 45, 477-501. DOI: 10.1023/A:1023967026413

\begin{tabular}{l} 
Autor \\
\hline Romero-Martín, Rosario (rromero@unizar.es). \\
Profesora en la Universidad de Zaragoza. Es la autora de contacto para \\
este artículo. Sus líneas de investigación se centran en la enseñanza de la \\
actividad física, la expresión corporal y las emociones. Su dirección postal \\
es Ronda Misericordia, 5. 22001 Huesca (España)
\end{tabular}
es Ronda Misericordia, 5. 22001 Huesca (España)
To know more / Saber más

ORCID

$\underline{0000-0002-5019-4574}$

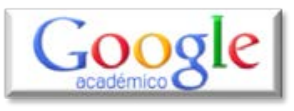

ResearchGate

Castejón-Oliva, Francisco Javier (javier.castejon@uam.es).

Sus líneas de investigación están dirigidas a la formación inicial del profesorado de educación física, la evaluación de los aprendizajes y la enseñanza del deporte. Tiene diversas publicaciones sobre el tema de evaluación del alumnado en la educación superior. Su dirección postal es Universidad Autónoma de Madrid. Calle Francisco Tomás y Valiente, 3. Ciudad Universitaria de Cantoblanco. 28049 Madrid

\section{López-Pastor, Víctor (vlopez@mpc.uva.es).}

Principales líneas de investigación: Evaluación Formativa y Compartida en Educación Superior, Formación Inicial y Permanente del Profesorado, Evaluación Formativa en Educación Física. Su dirección postal es Universidad de Valladolid. Facultad de Educación de Segovia. Campus María Zambrano (Universidad de Valladolid). Pza/ Alto Leones de Castilla, 1. 40005-Segovia

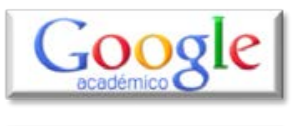

ResearchGate

\section{RELIEVE}

\section{Revista ELectrónica de Investigación y $\mathbf{E V}$ aluación Educativa E-Journal of Educational Research, Assessment and Evaluation}

[ISSN: 1134-4032]

(C) Copyright, RELIEVE. Reproduction and distribution of this articles it is authorized if the content is no modified and their origin is indicated (RELIEVE Journal, volume, number and electronic address of the document).

(C) Copyright, RELIEVE. Se autoriza la reproducción y distribución de este artículo siempre que no se modifique el contenido y se indique su origen (RELIEVE, volumen, número y dirección electrónica del documento). 